A. S. POZNYAK (México)

M. I. TAKSAR (Stony Brook, N.Y.)

\title{
ROBUST CONTROL OF LINEAR STOCHASTIC SYSTEMS WITH FULLY OBSERVABLE STATE
}

Abstract. We consider a multidimensional linear system with additive inputs (control) and Brownian noise. There is a cost associated with each control. The aim is to minimize the cost. However, we work with the model in which the parameters of the system may change in time and in addition the exact form of these parameters is not known, only intervals within which they vary are given. In the situation where minimization of a functional over the class of admissible controls makes no sense since the value of such a functional is different for different systems within the class, we should deal not with a single problem but with a family of problems. The objective in such a setting is twofold. First, we intend to establish existence of a state feedback linear robust control which stabilizes any system within the class. Then among all robust controls we find the one which yields the lowest bound on the cost within the class of all systems under consideration. We give the answer in terms of a solution to a matrix Riccati equation and we present necessary and sufficient conditions for such a solution to exist. We also state a criterion when the obtained bound on the cost is sharp, that is, the control we construct is actually a solution to the minimax problem.

1. Introduction. The classical stochastic control theory deals with a stochastic system in which the uncertainty is of exogenous type and is described by a stochastic process with known characteristics. In addition to the exogenous stochastic process the dynamics of such a system depends on the control functional (policy) which can be chosen within an a priori known class. Usually there is a cost associated with each control functional. The objective is to find the minimal cost and the minimizing (optimal)

1991 Mathematics Subject Classification: 60J10, 60B15, 49K15, $49 \mathrm{~J} 15$.

Key words and phrases: stochastic control, robust control, stochastic differential equations, Riccati equation. 
functional. By and large such problems are solved by dynamic programming methods and by analyzing the corresponding Bellman equation ([2], [5]).

However, there are a lot of applications in which the main uncertainty is not of a probabilistic type. It is of internal nature, lying with one's inability to determine explicitly the parameters of the system. Usually this uncertainty is specified by the intervals which the coefficients of the system belong to. Optimizing the value of the cost functional for the system whose parameters are unknown (and therefore the value to be optimized is unknown as well) makes no sense. Rather it is reasonable to look at the largest possible cost corresponding to different values of the system parameters. Thus one can seek the control which stabilizes the system for any values of the parameters and whose performance for the worst case scenario is the best. In other words, one looks for a control which is robust for a whole class of systems. This is where the notion of robust control comes from ([11], [3], [7], [12].)

In many dynamical systems, however, both of the above features are present. Namely, there are exogenous random disturbances as well as uncertainties in the parameters (the so-called mixed uncertainties [4], [6]). The novelty of this paper is in developing robust control for the systems with mixed uncertainties, which will be called stochastic robust control in the sequel. We consider the case with the exogenous stochastic process being Brownian motion and the state equation being linear with unknown time dependent coefficients. However, the intervals which the values of these coefficients belong to are known.

The cost associated with a control is given by an integral functional. As always due to the uncertainty in the state equation coefficients, one cannot find an unequivocal expression for the value of the cost functional for such a problem. Instead we consider the whole class of problems with state equation coefficients subject to the same constraints as in our original problem. We are able to identify the control within the class of linear state feedback strategies and calculate a bound on its performance. We also find the control with minimal bound. The obtained policy is determined via a nonnegative solution of an algebraic matrix Riccati equation. We use the results of [10] to give necessary and sufficient conditions for existence of such a solution. These conditions are described in terms of data of the problem and allow one to identify those problems for which linear state feedback stochastic robust control can be constructed.

In the next section we introduce the basic definitions and formulate the main result. We also give a bound on the cost. Our main tools are martingale techniques, stochastic integration and algebraic matrix Riccati equations. In the last section we study the criterion for existence of a nonnegative solution to the matrix Riccati equation. This enables us to specify 
those sets of data for which one can guarantee existence of a robust control. We also present a condition on "sharpness" of the upper bound obtained in the previous section.

2. Basic notions and formulation of the main result. We start with a probability space $\left(\Omega, \mathcal{F}_{t}, \mathrm{P}\right)$ and a $q$-dimensional $\mathcal{F}_{t}$-adapted Brownian motion $w(t)$ on this space. The dynamics of the system under control is given by the following stochastic differential equation:

$$
\begin{aligned}
d x(t) & =\left[A(t) x(t)+B_{1}(t) u(t)\right] d t+B_{2}(t) d w(t), \\
z(t) & =C(t) x(t), \quad t \geq 0, \\
x(0) & =x_{0} \in \mathbb{R}^{n} .
\end{aligned}
$$

Here

- $x(t) \in \mathbb{R}^{n}$ is the state of the system at time $t \in \mathbb{R}_{+}:=\{t: t \geq 0\}$,

- $x_{0}$ is a known initial position,

- $\{u(t)\}_{t \in \mathbb{R}_{+}} \in \mathbb{R}^{m}$ is an $\mathcal{F}_{t^{-}}$-adapted stochastic process called a policy, which represents the controllable input into the system,

- $z(t) \in \mathbb{R}^{r}$ is the output, characterizing the current value of the performance index,

- $A(t) \in \mathbb{R}^{n \times n}, B_{1}(t) \in \mathbb{R}^{n \times m}, B_{2}(t) \in \mathbb{R}^{n \times q}, C(t) \in \mathbb{R}^{r \times n}$ are realvalued matrix functions defined for any $t \geq 0$,

- $\{w(t)\}_{t \in \mathbb{R}_{+}}$is a $q$-dimensional Brownian motion which plays a role of random disturbance input into the system and for which

$$
[w(t)-w(s)] \sim \mathcal{N}(0, \Theta(t-s)), \quad \Theta=\Theta^{T}>0 .
$$

Let $A_{0} \in \mathbb{R}^{n \times n}, B_{10} \in \mathbb{R}^{n \times m}, \Delta_{A} \in \mathbb{R}^{n \times n}, \Delta_{B_{1}} \in \mathbb{R}^{n \times n}, \mathcal{B}_{2} \in \mathbb{R}^{n \times n}$, $\mathcal{C}_{0}, \mathcal{C} \in \mathbb{R}^{n \times n}, \eta_{A} \in \mathbb{R}^{n \times n}, \eta_{B_{1}} \in \mathbb{R}^{n \times n}\left(\eta_{A}=\eta_{A}^{T}>0, \eta_{B_{1}}=\eta_{B_{1}}^{T}>0\right)$ be given. Let $\mathcal{K}$ be the class of 4 -tuples of matrix functions defined below:

$$
\begin{aligned}
\mathcal{K}=\left\{\left(A(t), B_{1}(t), B_{2}(t), C(t)\right):\right. & A(t)=A_{0}+\Delta A(t), \forall t \\
& \Delta A(t) \eta_{A} \Delta A(t)^{T} \leq \Delta_{A}, \\
& B_{1}(t)=B_{10}+\Delta B_{1}(t), \\
& \Delta B_{1}(t) \eta_{B_{1}} \Delta B_{1}(t)^{T} \leq \Delta_{B_{1}}, \\
& B_{2}(t) B_{2}(t)^{T} \leq \mathcal{B}_{2}, \\
& \left.0<\mathcal{C}_{0} \leq C(t)^{T} C(t) \leq \mathcal{C}\right\} .
\end{aligned}
$$

The matrix functions $A(t), B_{1}(t), B_{2}(t), C(t)$ in the equations (1), (2) are not known. It is only assumed that they are measurable functions of $t$ and $\left(A(t), B_{1}(t), B_{2}(t), C(t)\right) \in \mathcal{K}$. With each such system and each policy 
$\{u(t)\}_{t \in \mathbb{R}_{+}}$we associate the costs

$$
\begin{aligned}
& J_{x_{0}}^{\mathrm{a}}(u):=\limsup _{T \rightarrow \infty} \mathrm{E}\left\{\frac{1}{T} \int_{0}^{T}\left[z(t)^{T} z(t)+u(t)^{T} \Lambda u(t)\right] d t\right\}, \\
& J_{x_{0}}^{\mathrm{p}}(u):=\limsup _{T \rightarrow \infty} \frac{1}{T} \int_{0}^{T}\left[z(t)^{T} z(t)+u(t)^{T} \Lambda u(t)\right] d t .
\end{aligned}
$$

Here $\Lambda=\Lambda^{T}>0$ is an a priori given constant matrix which characterizes weights of control actions in the general cost (5) and $\mathrm{E}\{\cdot\}$ is the expectation operator with respect to the measure $\mathrm{P}$.

Our aim is to minimize the maximum (or supremum) of this cost functional over the class of problems with matrix function coefficients belonging to the class $\mathcal{K}$. However, in this minimax problem we will consider not all policies $\{u(t)\}_{t \in \mathbb{R}_{+}}$but only those which at each moment $t \geq 0$ can be represented as a linear function of the state $x(t)$ of the system given by (1). (In this case the classical theory of stochastic differential equations [1] guarantees existence and uniqueness of an $\mathcal{F}_{t}$-adapted solution $x(t)$ to (1)). Such type of control will be called state feedback linear control.

In addition we require our policies to be stabilizing according to the definitions below.

Definition 1. A policy $\{u(t)\}_{t \in \mathbb{R}_{+}}$is called stabilizing on average if

$$
\limsup _{T \rightarrow \infty} \mathrm{E}\left\{\frac{1}{T} \int_{0}^{T} x(t)^{T} x(t) d t\right\}<\infty .
$$

Definition 2. A policy $\{u(t)\}_{t \in \mathbb{R}_{+}}$is called stabilizing almost surely if

$$
\limsup _{T \rightarrow \infty} \frac{1}{T} \int_{0}^{T} x(t)^{T} x(t) d t \stackrel{\text { a.s. }}{<} \infty .
$$

We denote by $\mathcal{U}_{\mathrm{a}}$ the class of all linear feedback policies by $\mathcal{U}$, the class of all linear feedback policies stabilizing on average, and by $\mathcal{U}_{\mathrm{p}}$ the class of all linear feedback stabilizing almost surely.

Define

$$
\begin{aligned}
& J_{\mathrm{a}}^{*}:=\inf _{\{u(t)\}_{t \in \mathbb{R}_{+} \in \mathcal{U}_{\mathrm{a}}}} \sup _{\mathcal{K}} J_{x_{0}}^{\mathrm{a}}(u), \\
& J_{\mathrm{p}}^{*}:=\inf _{\{u(t)\}_{t \in \mathbb{R}_{+}} \in \mathcal{U}_{\mathrm{p}}} \sup _{\mathcal{K}} J_{x_{0}}^{\mathrm{p}}(u) .
\end{aligned}
$$

The objective is:

- to obtain an upper bound on $J_{\mathrm{a}}^{*}$ and $J_{\mathrm{p}}^{*}$, i.e.

$$
J_{\mathrm{a}}^{*} \leq C_{1}(\Theta), \quad J_{\mathrm{p}}^{*} \stackrel{\text { a.s. }}{\leq} C_{2}(\Theta)
$$


- to find the conditions when it is sharp, i.e.

$$
J_{\mathrm{a}}^{*}=C_{1}(\Theta), \quad J_{\mathrm{p}}^{*} \stackrel{\text { a.s. }}{=} C_{2}(\Theta) .
$$

TheOrem 1. Suppose that

$$
R_{0}:=B_{10}^{T} \Lambda^{-1} B_{10}-\Delta_{A}-\Delta_{B_{1}}>0 .
$$

If there exists a symmetric nonnegative matrix solution $P=P^{T} \geq 0$ of the matrix Riccati equation

$$
P A_{0}+A_{0}^{T} P+P R_{0} P+Q_{0}=0
$$

where

$$
Q_{0}:=\eta_{A}^{-1}+\mathcal{C}
$$

then the policy

$$
u(t)=\bar{u}(t):=-\left(\Lambda+\eta_{B_{1}}^{-1}\right)^{-1} B_{10}^{T} P x(t)
$$

is stabilizing on average and almost surely. In addition, the following upper bounds hold:

$$
J_{\mathrm{a}}^{*} \leq C_{1}(\Theta), \quad J_{\mathrm{p}}^{*} \stackrel{\text { a.s. }}{\leq} C_{2}(\Theta)
$$

with

$$
C_{1}(\Theta)=C_{2}(\Theta)=\limsup _{T \rightarrow \infty} \frac{1}{2 T} \int_{0}^{T} \operatorname{tr}\left\{B_{2}(t) \Theta B_{2}(t)^{T} P\right\} d t .
$$

P r o of. Using Ito's formula we calculate the differential of the Lyapunov function $V(x)=x^{T} P x$ :

$$
\begin{aligned}
d V(x(t)) & \\
= & 2 x(t)^{T} P d x(t)+I(t) d t \\
= & 2 x(t)^{T} P\left[A_{0} x(t)+B_{10} u(t)\right] d t+I(t) d t \\
& +2 x(t)^{T} P B_{2}(t) d w(t)+2 x(t)^{T} P\left[\Delta A(t) x(t)+\Delta B_{1}(t) u(t)\right] d t
\end{aligned}
$$

where

$$
I(t):=\frac{1}{2} \operatorname{tr}\left\{B_{2}(t) \Theta B_{2}(t)^{T} P\right\} .
$$

The following matrix inequality is true for any matrices $X, Y$ and $\Lambda=\Lambda^{T}>0$ :

$$
X^{T} Y+Y^{T} X \leq X^{T} \Lambda X+Y^{T} \Lambda^{-1} Y .
$$

From (15) we get (the matrix $\Lambda_{0}$ below will be chosen later)

$$
\begin{aligned}
2 x(t)^{T} P \Delta A(t) x(t) & =2\left(\Delta A(t)^{T} P x(t), x(t)\right) \\
& \leq x(t)^{T}\left[P \Delta A(t) \eta_{A} \Delta A(t)^{T} P\right] x(t)+x(t)^{T} \eta_{A}^{-1} x(t) \\
& \leq x(t)^{T}\left[P \Delta_{A} P+\eta_{A}^{-1}\right] x(t),
\end{aligned}
$$




$$
\begin{aligned}
2 x(t)^{T} P \Delta B_{1}(t) u(t) & =2\left(\Delta B_{1}(t)^{T} P x(t), u(t)\right) \\
& \leq x(t)^{T} P \Delta B_{1}(t) \eta_{B_{1}} \Delta B_{1}(t)^{T} P x(t)+u(t)^{T} \eta_{B_{1}}^{-1} u(t) \\
& \leq x(t)^{T} P \Delta_{B_{1}} P x(t)+u(t)^{T} \eta_{B_{1}}^{-1} u(t),
\end{aligned}
$$

and also

$$
\begin{aligned}
2 x(t)^{T} P A_{0} x(t)= & x(t)^{T}\left[P A_{0}+A_{0}^{T} P\right] x(t), \\
2 x(t)^{T} P B_{10} u(t)= & \left\|\Lambda_{0}^{-1 / 2} B_{10}^{T} P x(t)+\Lambda_{0}^{1 / 2} u(t)\right\|^{2} \\
& -x(t)^{T} P B_{10} \Lambda_{0}^{-1} B_{10}^{T} P x(t)-u(t)^{T} \Lambda_{0} u(t) .
\end{aligned}
$$

Substituting these relations into (14), we derive

$$
\begin{aligned}
d V(x(t))= & 2 x(t)^{T} P d x(t)+I(t) d t \\
\leq & x(t)^{T}\left[P A_{0}+A_{0}^{T} P+P R_{0} P+Q_{0}\right] x(t) d t+I(t) d t \\
& +\left\|\Lambda_{0}^{-1 / 2} B_{10}^{T} P x+\Lambda_{0}^{1 / 2} u\right\|^{2} d t+2 x(t)^{T} P B_{2}(t) d w(t) \\
& -\|z(t)\|^{2} d t+u(t)^{T}\left[\eta_{B_{1}}^{-1}-\Lambda_{0}\right] u(t) d t .
\end{aligned}
$$

Choose $\Lambda_{0}:=\eta_{B_{1}}^{-1}+\Lambda$. Then, in view of (9), in (16) we have

$$
x(t)^{T}\left(P A_{0}+A_{0}^{T} P+P R_{0} P+Q_{0}\right) x(t)=0 .
$$

Also, the choice of the matrix $\Lambda_{0}$ and the control (11) implies

$$
\left\|\Lambda_{0}^{-1 / 2} B_{10}^{T} P x+\Lambda_{0}^{1 / 2} u\right\|^{2}=0 .
$$

Hence,

$$
d V(x(t)) \leq I(t) d t+2 x(t)^{T} P B_{2}(t) d w(t)-\|z(t)\|^{2} d t-u(t)^{T} \Lambda u(t) d t .
$$

Integrating (17) from 0 to $T$ and taking into account that $V(x(T)) \geq 0$, we deduce

$$
\begin{aligned}
& \frac{1}{T} \int_{0}^{T}\left[\|z(t)\|^{2}+u(t)^{T} \Lambda u(t)\right] d t \\
& \quad \leq \frac{1}{T} \int_{0}^{T} I(t) d t+\frac{1}{T} \int_{0}^{T} 2 x(t)^{T} P B_{2}(t) d w(t)-\frac{1}{T} V(x(T))+\frac{1}{T} V(x(0)) \\
& \quad \leq \frac{1}{T} \int_{0}^{T} I(t) d t+\frac{1}{T} \int_{0}^{T} 2 x(t)^{T} P B_{2}(t) d w(t)+\frac{1}{T} V(x(0))
\end{aligned}
$$

Hence,

$$
\limsup _{T \rightarrow \infty} \mathrm{E}\left\{\frac{1}{T} \int_{0}^{T}\left[\|z(t)\|^{2}+u(t)^{T} \Lambda u(t)\right] d t\right\} \leq \limsup _{T \rightarrow \infty} \frac{1}{T} \int_{0}^{T} I(t) d t
$$


and

$$
\limsup _{T \rightarrow \infty} \frac{1}{T} \int_{0}^{T}\left[\|z(t)\|^{2}+u(t)^{T} \Lambda u(t)\right] d t \stackrel{\text { a.s. }}{\leq} \limsup _{T \rightarrow \infty} \frac{1}{T} \int_{0}^{T} I(t) d t+I_{0},
$$

where

$$
I_{0}:=\limsup _{T \rightarrow \infty} \frac{1}{T} \int_{0}^{T} 2 x(t)^{T} P B_{2}(t) d w(t) .
$$

Inequalities (19)-(20) imply that our policy is stabilizing on average and

$$
J_{\mathrm{a}}^{*} \leq C_{1}(\Theta), \quad J_{\mathrm{p}}^{*} \stackrel{\text { a.s. }}{\leq} C_{2}(\Theta)+I_{0} .
$$

To conclude the proof we need to show

$$
I_{0} \stackrel{\text { a.s. }}{=} 0 \text {. }
$$

First notice that from the inequality (19) and the assumption (4) it follows that

$$
\begin{aligned}
\limsup _{T \rightarrow \infty} \frac{1}{T} \int_{0}^{T} \mathrm{E}\left\{\|x(t)\|^{2}\right\} d t & \leq \lambda_{\min }^{-1}\left(\mathcal{C}_{0}\right) \limsup _{T \rightarrow \infty} \frac{1}{T} \int_{0}^{T} \mathrm{E}\left\{\|z(t)\|^{2}\right\} d t \\
& \leq \lambda_{\max }\left(\mathcal{C}_{0}\right)^{-1} \limsup _{T \rightarrow \infty} \frac{1}{T} \int_{0}^{T} I(t) d t<\infty
\end{aligned}
$$

(here $\lambda_{\min }(\cdot)$ and $\lambda_{\max }(\cdot)$ are the minimal and maximal eigenvalues of the relevant matrices). Therefore there exists a constant $k>0$ such that

$$
F(T):=\int_{0}^{T} \mathrm{E}\left\{\|x(t)\|^{2}\right\} d t \leq k(T+1) .
$$

Notice that

$$
\operatorname{tr}\left\{B_{2}(t) \Theta B_{2}(t)^{T} P\right\} \leq \lambda_{\max }(\Theta) \operatorname{tr}\left\{\mathcal{B}_{2} P\right\}=\text { const }<\infty .
$$

Hence, we can conclude from (19), (21), (23) that $J_{\mathrm{a}}^{*} \leq$ const $<\infty$ uniformly in the class $\mathcal{K}$. Thus the policy $\{\bar{u}(t)\}_{t \in \mathbb{R}_{+}}$is stabilizing on average, i.e., $\{\bar{u}(t)\}_{t \in \mathbb{R}_{+}} \in \mathcal{U}_{\mathrm{a}}$.

Now consider

$$
\begin{aligned}
\int_{0}^{T}(t+1)^{-2} \mathrm{E}\left\{\|x(t)\|^{2}\right\} d t & =\int_{0}^{T}(t+1)^{-2} d F(t) \\
& =(T+1)^{-2} F(T)+2 \int_{0}^{T}(t+1)^{-3} F(t) d t .
\end{aligned}
$$

In view of (24) the right hand side of (25) does not exceed 


$$
k(T+1)^{-1}+2 k \int_{0}^{T}(t+1)^{-2} d t
$$

Letting $T \rightarrow \infty$ yields

$$
\int_{0}^{\infty}(t+1)^{-2} \mathrm{E}\left\{\|x(t)\|^{2}\right\} d t<\infty
$$

Define the process

$$
S(t):=\frac{1}{t} \int_{0}^{t} 2 x(\tau)^{T} P B_{2}(\tau) d w(\tau)
$$

and consider the sequence $s_{n}:=S(n)$. Note that

$$
\begin{aligned}
s_{n}= & \left(\frac{n-1}{n}\right) \frac{1}{n-1}\left[\int_{0}^{n-1} 2 x(\tau)^{T} P B_{2}(\tau) d w(\tau)\right. \\
& \left.+\int_{n-1}^{n} 2 x(\tau)^{T} P B_{2}(\tau) d w(\tau)\right] \\
= & \left(1-\frac{1}{n}\right) s_{n-1}+\frac{1}{n} \int_{n-1}^{n} 2 x(\tau)^{T} P B_{2}(\tau) d w(\tau) .
\end{aligned}
$$

Therefore

$$
\begin{aligned}
\mathrm{E}\left\{s_{n}^{2} \mid \mathcal{F}_{n-1}\right\} \stackrel{\text { a.s. }}{\leq} & \left(1-\frac{1}{n}\right) s_{n-1}^{2} \\
& +\frac{4}{n^{2}} \int_{n-1}^{n} \mathrm{E}\left\{x(\tau)^{T} P B_{2}(\tau) \Theta B_{2}(\tau)^{T} P x(\tau) \mid \mathcal{F}_{n-1}\right\} d \tau,
\end{aligned}
$$

where $\mathcal{F}_{n-1}=\sigma(w(\tau), \tau \leq n-1)$. Notice that

$$
\sum_{n=1}^{\infty} \frac{1}{n^{2}} \int_{n-1}^{n} \mathrm{E}\left\{x(\tau)^{T} P B_{2}(\tau) \Theta B_{2}(\tau)^{T} \operatorname{Px}(\tau) \mid \mathcal{F}_{n-1}\right\} d \tau \stackrel{\text { a.s. }}{<} \infty .
$$

if

$$
\sum_{n=1}^{\infty} \frac{1}{n^{2}} \int_{n-1}^{n} \mathrm{E}\left\{x(\tau)^{T} P B_{2}(\tau) \Theta B_{2}(\tau)^{T} P x(\tau)\right\} d \tau<\infty .
$$

Since

$$
\begin{aligned}
\sum_{n=1}^{\infty} \frac{1}{n^{2}} \int_{n-1}^{n} \mathrm{E}\{ & \left.x(\tau)^{T} P B_{2}(\tau) \Theta B_{2}(\tau)^{T} P x(\tau)\right\} d \tau \\
& \leq \sup _{t} \lambda_{\max }\left(P B_{2}(t) \Theta B_{2}(t)^{T} P\right) \sum_{n=1}^{\infty} \frac{1}{n^{2}} \int_{n-1}^{n} \mathrm{E}\left\{\|x(\tau)\|^{2}\right\} d \tau
\end{aligned}
$$




$$
\begin{aligned}
& \leq \text { const } \sum_{n=1}^{\infty}\left(1+\frac{1}{n}\right)^{2} \frac{1}{(n+1)^{2}} \int_{n-1}^{n} \mathrm{E}\left\{\|x(\tau)\|^{2}\right\} d \tau \\
& \leq \operatorname{const} \sum_{n=1}^{\infty} 4 \int_{n-1}^{n} \frac{1}{(\tau+1)^{2}} \mathrm{E}\left\{\|x(\tau)\|^{2}\right\} d \tau \\
& =4 \text { const } \int_{0}^{\infty} \frac{1}{(\tau+1)^{2}} \mathrm{E}\left\{\|x(\tau)\|^{2}\right\} d \tau,
\end{aligned}
$$

inequality (28) follows from (26).

Let us now use the following Robbins-Siegmund lemma (see [8]).

LEMma 1. Let $\left\{\mathcal{F}_{n}\right\}$ be a sequence of $\sigma$-algebras and $x_{n}(\omega), \alpha_{n}(\omega), \beta_{n}(\omega)$ and $\xi_{n}(\omega)$ be $\mathcal{F}_{n}$-measurable nonnegative random variables on a probability space $(\Omega, \mathcal{F}, \mathrm{P})$ such that for all $n=1,2, \ldots, \mathrm{E}\left\{x_{n+1}(\omega) \mid \mathcal{F}_{n}\right\}$ exists and

$$
\mathrm{E}\left\{x_{n+1}(\omega) \mid \mathcal{F}_{n}\right\} \leq x_{n}(\omega)\left(1+\alpha_{n}(\omega)\right)+\beta_{n}(\omega)-\xi_{n}(\omega)
$$

with probability 1 . Then, for all $\omega \in \Omega_{0}$, where

$$
\Omega_{0}=\left\{\omega \in \Omega \mid \sum_{n=1}^{\infty} \alpha_{n}(\omega)<\infty, \sum_{n=1}^{\infty} \beta_{n}(\omega)<\infty\right\}
$$

the limit $\lim _{n \rightarrow \infty} x_{n}(\omega)=x^{*}(\omega)$ exists and

$$
\sum_{n=1}^{\infty} \xi_{n}(\omega)<\infty
$$

The lemma implies the existence of $s^{*} \stackrel{\text { a.s. }}{<} \infty$ such that $\lim _{n \rightarrow \infty} s_{n}=s^{*}$. In addition, the same lemma yields

$$
\sum_{n=1}^{\infty} \frac{1}{n} s_{n-1}^{2} \stackrel{\text { a.s. }}{<} \infty .
$$

Since the harmonic series is divergent, there exists a subsequence $n_{k}$ (maybe depending on $\omega \in \Omega$ ) such that $\lim _{k \rightarrow \infty} s_{n_{k}}=0$. Hence

$$
s^{*} \stackrel{\text { a.s. }}{=} 0 \text {. }
$$

Now we prove that

$$
\lim _{t \rightarrow \infty} S(t) \stackrel{\text { a.s. }}{=} 0 .
$$

Put 


$$
\begin{aligned}
\zeta_{n}:= & \sup _{t \in[n, n+1)}\left|S(t)-s_{n}\right| \\
= & \sup _{t \in[n, n+1)} \mid \frac{1}{n} \int_{n}^{t} 2 x(\tau)^{T} P B_{2}(\tau) d w(\tau) \\
& +\left(\frac{1}{t}-\frac{1}{n}\right) \int_{0}^{t} 2 x(\tau)^{T} P B_{2}(\tau) d w(\tau) \mid .
\end{aligned}
$$

Obviously,

$$
\begin{aligned}
\zeta_{n} \leq & \sup _{t \in[n, n+1)}\left|\frac{1}{n} \int_{n}^{t} 2 x(\tau)^{T} P B_{2}(\tau) d w(\tau)\right| \\
& +\frac{1}{n^{2}} \sup _{t \in[n, n+1)}\left|\int_{0}^{t} 2 x(\tau)^{T} P B_{2}(\tau) d w(\tau)\right| .
\end{aligned}
$$

Thus,

$$
\begin{aligned}
\mathrm{P}\left\{\zeta_{n} \geq \varepsilon\right\} \leq & \varepsilon^{-2} \mathrm{E}\left\{\zeta_{n}^{2}\right\} \\
\leq & 2 \varepsilon^{-2}\left(\frac{1}{n^{2}} \mathrm{E}\left\{\left[\sup _{t \in[n, n+1)}\left|\int_{n}^{t} 2 x(\tau)^{T} P B_{2}(\tau) d w(\tau)\right|\right]^{2}\right\}\right. \\
& \left.+\frac{1}{n^{4}} \mathrm{E}\left\{\left[\sup _{t \in[0, n+1)}\left|\int_{0}^{t} 2 x(\tau)^{T} P B_{2}(\tau) d w(\tau)\right|\right]^{2}\right\}\right) .
\end{aligned}
$$

By (24) and Doob's inequality (see [9]) the right hand side of (31) does not exceed (below const stands for a generic constant whose value may differ in different lines)

$$
\begin{aligned}
8 \varepsilon^{-2}\left(\frac{1}{n^{2}} \mathrm{E}\left\{\int_{n}^{n+1} 4 x(\tau)^{T} P B_{2}(\tau) \Theta B_{2}(\tau)^{T} P x(t) d \tau\right\}\right. & \\
& \left.+\frac{1}{n^{4}} \mathrm{E}\left\{\int_{0}^{n+1} 4 x(\tau)^{T} P B_{2}(\tau) \Theta B_{2}(\tau)^{T} P x(t) d \tau\right\}\right) \\
\leq & \varepsilon^{-2} \text { const }\left[\frac{1}{n^{2}} \int_{n}^{n+1} \mathrm{E}\left\{\|x(\tau)\|^{2}\right\} d \tau+\frac{1}{n^{4}} \int_{0}^{n+1} \mathrm{E}\left\{\|x(\tau)\|^{2}\right\} d \tau\right] \\
= & \varepsilon^{-2} \text { const }\left[\frac{1}{n^{2}} \int_{n}^{n+1} \mathrm{E}\left\{\|x(\tau)\|^{2}\right\} d \tau+\frac{1}{n^{4}} F(n+1)\right] \\
\leq & \varepsilon^{-2} \text { const }\left[\left(1+\frac{2}{n}\right)^{2} \frac{1}{(n+2)^{2}} \int_{n}^{n+1} \mathrm{E}\left\{\|x(\tau)\|^{2}\right\} d \tau+\frac{1}{n^{4}} k(n+2)\right]
\end{aligned}
$$




$$
\begin{aligned}
& \leq \varepsilon^{-2} \text { const }\left[\frac{1}{(n+2)^{2}} \int_{n}^{n+1} \mathrm{E}\left\{\|x(\tau)\|^{2}\right\} d \tau+\frac{1}{n^{3}}\right] \\
& \leq \varepsilon^{-2} \text { const }\left[\int_{n}^{n+1} \frac{1}{(\tau+1)^{2}} \mathrm{E}\left\{\|x(\tau)\|^{2}\right\} d \tau+\frac{1}{n^{3}}\right] .
\end{aligned}
$$

Therefore,

$$
\begin{aligned}
\sum_{n=1}^{\infty} \mathbf{P}\left\{\zeta_{n} \geq \varepsilon\right\} & \leq \varepsilon^{-2} \text { const } \sum_{n=1}^{\infty}\left[\int_{n}^{n+1} \frac{1}{(\tau+1)^{2}} \mathrm{E}\left\{\|x(\tau)\|^{2}\right\} d \tau+\frac{1}{n^{3}}\right] \\
& =\varepsilon^{-2} \text { const }\left[\int_{1}^{\infty} \frac{1}{(\tau+1)^{2}} \mathrm{E}\left\{\|x(\tau)\|^{2}\right\} d \tau+\sum_{n=1}^{\infty} \frac{1}{n^{3}}\right]<\infty
\end{aligned}
$$

by (26). The Borel-Cantelli lemma implies $\lim _{n \rightarrow \infty} \zeta_{n} \stackrel{\text { a.s. }}{=} 0$. This together with (29) yields (30).

Since $\lim _{t \rightarrow \infty} S(t)=I_{0}$ we have proved (20). In view of assumption (4), inequality (20) and equality (22) we conclude that the policy $\{\bar{u}(t)\}_{t \in \mathbb{R}_{+}}$ given by (11) is stabilizing almost surely, i.e., $\{\bar{u}(t)\}_{t \in \mathbb{R}_{+}} \in \mathcal{U}_{\mathrm{p}}$.

\section{Existence of a solution to the Riccati equation. Sharpness} of the bound. The following theorem provides a criterion for existence of a nonnegative solution for the algebraic matrix Riccati equation of the previous section.

Theorem 2. Suppose $A_{0} \in \mathbb{R}^{n \times n}$ and $0<R_{0}=R_{0}^{T} \in \mathbb{R}^{n \times n}$ and $0 \leq Q_{0}=Q_{0}^{T} \in \mathbb{R}^{n \times n}$ are defined by (8) and (10). Let the pair $\left(A_{0}, R_{0}^{1 / 2}\right)$ be stabilizable and the pair $\left(Q_{0}^{1 / 2}, A_{0}\right)$ be observable. Then the matrix Riccati equation (9)

$$
P A_{0}+A_{0}^{T} P+P R_{0} P+Q_{0}=0
$$

has a unique positive solution $0<P=P^{T}$ if and only if the frequency condition

$$
I-R_{0}{ }^{1 / 2}\left[-i \omega I+A_{0}^{T}\right]^{-1} Q_{0}\left[i \omega I+A_{0}\right]^{-1} R_{0}{ }^{1 / 2} \geq 0
$$

is satisfied for any $\omega \in(-\infty, \infty), \omega \neq \omega_{j}$, where $i \omega_{j}(j=1, \ldots, N)$ are the eigenvalues of $A_{0}$.

This theorem is a direct consequence of Lemma 5 of [10].

TheOREm 3. The upper bound (12) is sharp (attainable) if $\emptyset \neq \mathcal{K}_{0} \subseteq \mathcal{K}$, where 


$$
\begin{aligned}
\mathcal{K}_{0}:=\{ & \left(A\left(t_{0}\right), B_{1}\left(t_{0}\right), B_{2}\left(t_{0}\right), C\left(t_{0}\right)\right): \eta_{A} \Delta A^{T}\left(t_{0}\right) P=I, \\
& \Delta A\left(t_{0}\right) \eta_{A} \Delta A^{T}\left(t_{0}\right)=\Delta_{A}, \quad \eta_{B_{1}} \Delta B_{1}^{T}\left(t_{0}\right)=\left[\Lambda+\eta_{B_{1}}^{-1}\right]^{-1} B_{10}^{T}, \\
& \Delta B_{1}\left(t_{0}\right) \eta_{B_{1}} \Delta B_{1}^{T}\left(t_{0}\right)=\Delta_{B_{1}}, \quad C^{T}\left(t_{0}\right) C\left(t_{0}\right)=\mathcal{C}, \\
& \left.\mathcal{B}_{2}^{1 / 2}\left(\mathcal{B}_{2}^{1 / 2}\right)^{T}=\mathcal{B}_{2}, \quad B_{2}\left(t_{0}\right)=\mathcal{B}_{2}^{1 / 2} U, \quad \text { for a unitary matrix } U\right\} .
\end{aligned}
$$

Proof. The matrix inequality (15) is tight if $Y=\Lambda X$. Repeating the proof of Theorem 1 with inequalities replaced by equalities we get the statement of Theorem 2 .

\section{References}

[1] L. Arnold, Stochastic Differential Equations: Theory and Applications, Wiley, New York, 1974.

[2] W. H. Fleming and R. Rishel, Deterministic and Stochastic Optimal Control, Springer, New York, 1975.

[3] B. A. Francis, A Course in $H^{\infty}$ Control Theory, Lecture Notes in Control and Inform. Sci., Springer, New York, 1987.

[4] K. Glover and D. Mustafa, Derivation of the maximum entropy $H_{\infty}$-controller and a state-space formula for its entropy, Internat. J. Control 50 (1989), 899-916.

[5] N. V. Krylov, Controlled Diffusion Processes, Springer, New York, 1980.

[6] A. P. Kurdyukov and A. S. Poznyak, Sensitivity of $H_{\infty}$-functionals to internal perturbations in controllable linear systems, Avtomat. i Telemekh. 1993 (4), 128136 (in Russian).

[7] A. Shiryayev, Probability, Springer, New York, 1984.

[8] I. P. Petersen and C. V. Hollot, A Riccati equation approach to the stabilization of uncertain linear systems, Automatica 22 (1986), 397-411.

[9] H. Robbins and D. Siegmund, A convergence theorem for nonnegative almost supermartingales and some applications, in: Optimizing Methods in Statistics, J. S. Rustagi (ed.), Academic Press, New York, 1971.

[10] J. C. Willems, Least squares stationary optimal control and algebraic Riccati equation, IEEE Trans. Automat. Control AC-16 (1971), 621-634.

[11] G. Zames, Feedback and optimal sensitivity: Model reference transformations, multiplicative seminorms and approximate inverses, ibid. AC-26 (1981), 301-320.

[12] K. Zhou and P. P. Karganekar, Robust stabilization of linear systems with norm bounded time varying uncertainty, Systems Control Lett. 10 (1988), 17-20.

Alexander S. Poznyak

CINVESTAV-IPN

Seccion de Control Automatico

Av. IPN 2508, esq. Calz. Ticoman, A.P. 14-740

Mexico 07000, Mexico

E-mail: apoznyak@ctrl.cinvestav.mx
M. I. Taksar

Department of Applied Mathematics

SUNY

Stony Brook, New York 11794-3600

U.S.A.

E-mail: taksar@ams.sunysb.edu

Received on 22.3.1995;

revised version on 12.1.1996 